\title{
Dislocation mutual interactions mediated by mobile impurities and the conditions for plastic instabilities
}

\author{
Fabio Leoni \\ School of Mechanical Engineering, Tel-Aviv University, Tel-Aviv 69978, Israel \\ Stefano Zapperi \\ CNR-IENI, Via R. Cozzi 53, 20125 Milano, Italy \\ ISI Foundation, Via Alassio 11C, 10126 Torino, Italy
}

\begin{abstract}
Matallic alloys, such as $\mathrm{Al}$ or $\mathrm{Cu}$, or mild steel, display plastic instabilities in a well defined range of temperatures and deformation rates, a phenomenon known as the Portevin-Le Chatelelier (PLC) effect. The stick-slip behavior, or serration, typical of this effect is due to the discontinuous motion of dislocations as they interact with solute atoms. Here we study a simple model of interacting dislocations and show how the classical Einstein fluctuation-dissipation relation can be used to define the temperature in a range of model parameters and to construct a phase diagram of serration that can be compared to experimental results. Furthermore, performing analytical calculations and numerically integrating the equations of motion, we clarify the crucial role played by dislocation mutual interactions in serration.
\end{abstract}

\section{INTRODUCTION}

Dislocation dynamics is a complex intermittent phenomenon involving the collective motion of many dislocations interacting between each other as well with obstacles eventually present in the material, like solute atoms or quenched dislocations from other glide planes [1 $[3]$. The long range stress produced by dislocations may lead to jamming and avalanche-like phenomena even in the absence of obstacles [4]. The presence of obstacles changes the local properties of the host material, resulting in a pinning force on nearby dislocations [5, [6]. Usually, this source of disorder for dislocations is taken to be quenched, so that its properties do not change within the relevant timescales of the system [7]. However, under specific conditions, the mobility of solute atoms in metallic alloys [8, 9], or oxygen vacancies in superconductors [10], plays an important role in the dynamics of these systems.

Here we are interested in studying the dynamics of interacting dislocations mediated by mobile impurities. The interplay between dislocations mutual interactions and pinning by mobile impurities is believed to be at the origin of plastic instabilities observed in metallic alloys under suitable loading conditions and temperature. One of the best studied forms of instability propagation is the Portevin-Le Chatelier (PLC) effect [1]. When a specimen of a dilute alloy (such as $\mathrm{Al}$ or $\mathrm{Cu}$ alloy, or mild steel) is strained in uniaxial loading, the mechanical response is often discontinuous. In constant applied strain rate tests, the stress versus strain (or time, which is proportional to strain) curves exhibit a succession of stress drops and reloading sequences (serration).

From a dynamical point of view, the jerky or stick-slip behavior of stress is related to the discontinuous motion of dislocations, namely, the pinning (stick) and unpinning (slip) of dislocations. The classical explanation of the PLC effect is via the dynamic strain aging (DSA) concept [12]. It is based on the interplay between the diffusivity of solute atoms and dislocations that can be arrested temporarily at obstacles during their waiting time. Thus, the longer the dislocations are arrested, the larger will be the stress required to unpin them. As a result, when the contribution from aging is large enough, the critical stress to move a dislocation increases with increasing waiting time or decreasing imposed strain rate. When these dislocations are unpinned, they move at large speed until they are arrested again. At high strain rates (or low temperatures), the time available for solute atoms to diffuse towards the dislocations in order to age them decreases and hence the stress required to unpin them decreases. Thus, in a range of strain rates and temperatures where these two time scales are of the same order of magnitude, the PLC instability manifests. The competition between the slow rate of aging and sudden unpinning of the dislocations, translates at the macroscopic level, into a negative strain rate sensitivity (SRS) of the flow stress as a function of strain rate. This basic instability mechanism, used in most phenomenological models for the PLC effect [13], is based on the behavior of individual dislocation and thus does not explain how dislocation motion can synchronize to yield macroscopic strain bursts.

Current theoretical approaches to models the PLC effect are based on a mesoscopic descriptive level (where coarse grained dislocation density is considered) in which phenomenological parameters are needed to construct the relative dynamical equations 13]. Modeling plastic deformation phenomena taking into account inhomogeneity at the dislocation level offers fundamental advantages compared to continuum mechanics approaches. The discrete dislocation dynamical (DDD) approaches allow, for example, to account for the intrinsic length scales, such as the grain size, the mesh length of a dislocation network or the cross-slip height, which is necessary to understand the formation of spatial dislocation structures, as persistent slip bands [14], and plastic instabilities, as Lüders band and the PLC effect 
[13]. The problem of spatial and temporal coupling in heterogeneously deforming materials, and the associated length and time scales to be included in constitutive laws, is a central issue in current attempts to bridge a gap between dislocation based constitutive models and continuum mechanics.

The general three-dimensional dynamical problem of dislocation lines interacting among each other is a complex problem due to the necessity to consider flexible lines conserving their connectivity and line length and take care of line interactions [15-21]. In several instances, however, dislocations are arranged into regular structures that are amenable to analytical treatment and more efficient simulation approach. Here we analyze the dynamics of the effective one-dimensional dislocation array called pileup interacting with mobile impurities. A slip band can be envisaged as a queue of dislocations, a pileup, pushed through a series of obstacles (solute atoms or immobile dislocations from other glide planes). In our case the obstacles perform a diffusive motion due to thermal effects, and interact with dislocations. This system can be viewed as a coupled one-dimensional channels of particles [22], in which particles in one channel (dislocations) are driven by an external force and experience a drag from the undriven particles (impurities) in the other channel. In the following we discuss before the single dislocation in a cloud of mobile impurities problem, analyzed in Ref.[23], and then we propose a generalization of the equations in the case of many interacting dislocations in a landscape of mobile impurities.

\section{SINGLE DISLOCATION INTERACTING WITH MOBILE IMPURITIES}

Recently the dynamics of a particle interacting with diffusing impurities in one dimension has been investigated by Laurson and Alava [23]. Despite the simplicity of the model, that makes it analytically tractable, it exhibits a rich dynamics. Here we describe this model as an introduction to the following section in which we will generalize the relative equations to the case of many interacting dislocations.

In the full formulation of the model discussed in Ref. [23], a particle in a cloud composed by a fixed number of $N_{p}$ impurities driven by an external force $F$ is considered. The force is given by $F=k(V t-x)$, where $V$ is the driving velocity and $k$ is a spring constant characterizing the response of the driving mechanism. The region of the parameter space is restricted to that in which the impurities have a vanishingly small probability to escape from the vicinity of the particle within the timescale of the simulation. Thus, the particle is dragging an impurity cloud with a fixed number of impurity particles without escaping from it. The equations of motion are

$$
\begin{aligned}
\mu \frac{\partial x}{\partial t} & =\sum_{i=1}^{N_{p}} f\left(x-x_{s, i}\right)+F, \\
\frac{\partial x_{s, i}}{\partial t} & =-f\left(x-x_{s, i}\right)+\eta_{i},
\end{aligned}
$$

where $x$ and $x_{s}$ are the position of the particle and the impurity particles, respectively. $f(z)$ is the interaction force between the particle and the impurity particle, $\mu$ defines the relative mobility of the impurity and the particle and $\eta_{i}$ are Gaussian white noise with standard deviation $\delta \eta$ and zero mean. The only condition imposed on the expression of the force $f(z)$ if $\left.\partial_{z} f(z)\right|_{z=0}=-f_{0}$. Here we are interested in particular on the behavior of the external force $F$, that in experiments represents the shear stress acting on dislocations. For $z=x-x_{s}$ close to zero, the following expression for the stochastic process $\partial_{t} F$ is derived in Ref. [23]

$$
\partial_{t}^{2} F=-k \partial_{t}^{2} x=-\left[\frac{k}{\mu}+\frac{f_{0}}{\mu}\left(N_{p}+\mu\right)\right] \partial_{t} F+\frac{k f_{0}}{\mu} \sum_{i=1}^{N_{p}} \eta_{i}+\frac{k f_{0}}{\mu}\left[V\left(N_{p}+\mu\right)-F\right] .
$$

Now, assuming that in the stationary state the last term in the r.h.s. of Eq2 has zero mean $\left(\left\langle V\left(N_{p}+\mu\right)-F\right\rangle=0\right)$ and that fluctuations are small compared to those of the white noise term $\left(\delta F \ll \sqrt{N_{p}} \delta \eta\right)$, the equation 2 reduces to the following Ornstein-Uhlenbeck process for $\partial_{t} F$

$$
\partial_{t}^{2} F=-\left[\frac{k}{\mu}+\frac{f_{0}}{\mu}\left(N_{p}+\mu\right)\right] \partial_{t} F+\frac{k f_{0}}{\mu} \sum_{i=1}^{N_{p}} \eta_{i}
$$

The condition of small fluctuations $\delta F \ll \sqrt{N_{p}} \delta \eta$ is fulfilled for most of the relevant parameter values condition; only for $k f_{0} \gg 1$ this is not the case. From Eq. 3 is possible to see that, after an initial transient, the system reaches the stationary state in which the external force $F$ fluctuates around a constant average value and these fluctuations are uncorrelated in time [23]. Therefore, the system composed by a single dislocation in a cloud of mobile impurities does not display a serration type behavior. 
The Eq. 2 can be solved exactly, without imposing conditions on fluctuations. Indeed is possible to rewrite it as a two dimensional (2d) Ornstein-Uhlenbeck process. Introducing the new variable $F^{*}=F-V\left(N_{p}+\mu\right)$ and considering $F^{*}$ and $\dot{F}^{*}$ as the components of a 2 d vector, the Eq. 2 can be written as a 2d Ornstein-Uhlenbeck process [24] for the vector variable $\left(F^{*}, \dot{F}^{*}\right)$

$$
\frac{d}{d t}\left(\begin{array}{l}
F^{*} \\
\dot{F}^{*}
\end{array}\right)=-\left(\begin{array}{lr}
0 & -1 \\
\omega_{0}^{2} & \gamma
\end{array}\right)\left(\begin{array}{l}
F^{*} \\
\dot{F}^{*}
\end{array}\right)+\left(\begin{array}{l}
0 \\
\Gamma(t)
\end{array}\right)
$$

where

$$
\begin{aligned}
\gamma & =\frac{1}{\mu}\left[k+f_{0}\left(N_{p}+\mu\right)\right], \\
\omega_{0}^{2} & =\frac{k f_{0}}{\mu} \\
\Gamma(t) & =\frac{k f_{0}}{\mu} \sum_{i=1}^{N_{p}} \eta_{i} .
\end{aligned}
$$

The solution for the average $\left\langle F^{*}\right\rangle$ is

$$
\left\langle F^{*}\right\rangle=\left.e^{-\gamma t}\right|_{11} F^{*}(0)+\left.e^{-\gamma t}\right|_{12} \dot{F}^{*}(0),
$$

where

$$
\begin{aligned}
& F^{*}(0)=F(0)-V\left(N_{p}+\mu\right)=-V\left(N_{p}+\mu\right), \\
& \dot{F}^{*}(0)=\dot{F}(0)=k V
\end{aligned}
$$

and the matrix $\gamma$ is

$$
\gamma=\left(\begin{array}{cr}
0 & -1 \\
\omega_{0}^{2} & \gamma
\end{array}\right)
$$

Diagonalizing the exponential matrix $e^{-\gamma t}$, we can write explicitly the expression of the average force $\langle F\rangle$ as

$$
\begin{aligned}
\langle F\rangle & =\left\langle F^{*}\right\rangle+V\left(N_{p}+\mu\right)=V\left(N_{p}+\mu\right)\left[1-\left.e^{-\gamma t}\right|_{11}\right]+\left.k V e^{-\gamma t}\right|_{12} \\
& =V\left(N_{p}+\mu\right)\left[1+\frac{\lambda_{1} e^{-\lambda_{2} t}-\lambda_{2} e^{-\lambda_{1} t}}{\lambda_{1}-\lambda_{2}}\right]+k V\left[\frac{e^{-\lambda_{1} t}-e^{-\lambda_{2} t}}{\left(\lambda_{1}-\lambda_{2}\right) t}\right] .
\end{aligned}
$$

where $\lambda_{1,2}$ are the eigenvalues of $\gamma$. The only case in which we can have fluctuations in the average force is obtained for $\left(\gamma^{2}-4 \omega_{0}^{2}\right)<0$, that leads to the following expression

$$
\langle F\rangle=V\left(N_{p}+\mu\right)\left\{1+e^{-\frac{\gamma}{2} t}\left[\frac{\gamma}{2} \frac{\sin \left(\sqrt{4 \omega_{0}^{2}-\gamma^{2}} t / 2\right)}{\sqrt{4 \omega_{0}^{2}-\gamma^{2}} / 2}-\cos \left(\sqrt{4 \omega_{0}^{2}-\gamma^{2}} t / 2\right)\right]\right\}-k V e^{-\frac{\gamma}{2} t} \frac{\sin \left(\sqrt{4 \omega_{0}^{2}-\gamma^{2}} t / 2\right)}{\sqrt{4 \omega_{0}^{2}-\gamma^{2}} t / 2}
$$

In this case oscillations (serration) emerges, but they decays exponentially fast. On the other hand, performing the stationary limit, one finds

$$
\lim _{t \rightarrow \infty}\langle F\rangle=V\left(N_{p}+\mu\right)
$$

Therefore, for any parameter values condition, a serration type behavior is not observed in the single dislocation in a cloud of mobile impurities model.

\section{DISLOCATION PILEUP INTERACTING WITH MOBILE IMPURITIES}

As the PLC effect is widely believed to be due to the dynamic interaction of dislocations with diffusing solute atoms, a natural formulation of the problem, in the framework of DDD approach, is to consider Eq. 1 for $N$ dislocations 
in a landscape of $N_{p}$ mobile impurities. To describe the dynamics of dislocations we use, as in [23], an overdamped equation, so that the velocity of a dislocation depends linearly on the resolved shear stress exerted on it [25]. Therefore, the equations of motion Eq. 1] are generalized as follows

$$
\begin{aligned}
\mu \frac{d x_{i}}{d t} & =G \sum_{\substack{(j \neq i) \\
j=1}}^{N} \frac{b_{i} b_{j}}{x_{i}-x_{j}}+b_{i} \sigma_{i}^{l}+\sum_{j=1}^{N_{p}} f_{P}\left(x_{i}-x_{s, j}\right) \\
\chi \frac{d x_{s, j}}{d t} & =-\sum_{i=1}^{N} f_{P}\left(x_{i}-x_{s, j}\right)+\eta_{j},
\end{aligned}
$$

where $G$ is the shear modulus, $b_{i}$ is the Burgers vector of the dislocation $i, \mu$ and $\chi$ are the damping constant of dislocations and impurities respectively. The external force $F$ is now explicitly indicated as the local shear stress $\sigma_{i}^{l}$ acting on each dislocation $i$, whose expression is

$$
\sigma_{i}^{l}=k\left[V t-\int_{0}^{t} b_{i} \frac{d x_{i}\left(t^{\prime}\right)}{d t^{\prime}} d t^{\prime}\right]=k\left[V t-b_{i}\left(x_{i}(t)-x_{i}(0)\right)\right],
$$

while for the pinning force $f_{P}(z)$, with $z=x_{i}-x_{s, j}$, and the noise term $\eta_{j}$ we have the expressions

$$
\begin{aligned}
f_{P}(z) & =-f_{0} \frac{z}{\xi_{P}} e^{-\left(z / \xi_{P}\right)^{2}} \\
\left\langle\eta_{j}(t)\right\rangle & =0 \\
\left\langle\eta_{j}(t) \eta_{j}\left(t^{\prime}\right)\right\rangle & =D \delta\left(t-t^{\prime}\right) \delta_{i j} .
\end{aligned}
$$

To emulate the behavior of a material in the bulk, we consider that $N$ point dislocations and $N_{p}$ impurities move along a line of size $L$ where periodic boundary conditions are chosen. In order to correctly take into account the effect of periodic boundary conditions, the interactions between dislocations are summed over theirs images 26.

We are interested in the total average stress exerted on dislocations, $\sigma=1 / N \sum_{i=1}^{N} \sigma_{i}^{l}$ (i.e. the external stress that is needed to apply on the material to obtain a constant strain rate). In view to study the behavior of the stress $\sigma$ in relation with the PLC effect, that is regulated principally by the temperature and strain rate as discussed in the introduction, we have imposed the relations $b_{i}=b=1, G=\mu=\xi_{P}=1, f_{0}=0.01$ and $k=0.1$ that fixes the time, space and force scales. The free parameters of the model are now $V, \chi, D$. In real materials impurities have already exerted aging effects over dislocations before the experiments (i. e. before that an external stress is imposed on the material). To take into account this effect the system is leaved to evolve without external stress $(k=0)$ for a waiting time $t_{w}$. The initial configuration of the system (at time $t=0$ ) consists of random distribution of dislocations and pinning centers. We choose $t_{w}=10^{6} \cdot d t=10^{4}$, where the integration step is $d t=10^{-2}$. This value of $t_{w}$ is sufficient for the system to stabilize its elastic energy during the initial part of the dynamics (for $t<t_{w}$ and $k=0$ ) [1] .

\section{THE CONDITIONS FOR SERRATION}

Before integrating numerically Eqs 12, 13, 14, we can obtain a set of necessary conditions for serration. First of all we can observe that if dislocations do not interact with any pinning center $\left(f_{P}(z)=0\right)$, the first equation of Eq12 do not posses normal modes of oscillation. This can be found employing a linear perturbative approach, as made in Ref. 27] to study the discrete cosmological N-body problem, or observing that the first equation of Eq[12 describes the so called Coulomb gas for the variables $x_{i}-(V / b) t$ at zero temperature [28].

If we now consider that dislocations interact with pinning centers $\left(f_{P}(z) \neq 0\right)$, but with the last ones quenched (that means $d x_{s, j} / d t=0$ ), from Eqs 12, 13, 14 we obtain

$$
\begin{aligned}
\mu \frac{d x}{d t} & =b \sigma+\frac{1}{N} \sum_{i=1}^{N} \sum_{j=1}^{N_{p}} f_{P}\left(x_{i}-x_{s, j}\right) \\
x_{s, j} & =c_{j}
\end{aligned}
$$

where $c_{j}$ are constants and the function $\Gamma$ can be obtained using the relation between $x_{i}$ and $\sigma_{i}^{l}$ in Eq. 13. Performing the ensemble average and the time integral on the relation to the right in Eq. 15] we obtain that $\langle\sigma\rangle=\mu V / b^{2}$ (so 
we do not have serration) if $\langle\Gamma\rangle=0$, that is if the constants $c_{j}$ do not correlate the variables $\sigma_{i}^{l}$ between them (i.e. if the $c_{j}$ do not depend in a specific way on the position of the variables $\left.x_{i}(0)\right)$. To find this result we employed the relation $\sum_{i=1(i \neq j)}^{N} \sum_{j=1}^{N} 1 /\left(x_{i}-x_{j}\right)=0$, introduced the variable $x=1 / N \sum_{i=1}^{N} x_{i}$ and considered the ensemble average respect to the $\left\{c_{j}\right\}$ configurations.

Another necessary condition to have serration can be found observing that in the range of parameters for which the impurities have a vanishingly small probability to escape from dislocations (the same case studied in Ref. [23] for the single dislocation problem) we can approximate the expression of the interacting force $f(z)$ for small $z$ as $f_{P}(z) \simeq f_{P}(0)+\left.z \partial_{z} f_{P}(z)\right|_{z=0}=-f_{0} z$. Employing this approximation, from Eqs 12, 13, 14, we find for $\sigma$ the following $2 \mathrm{~d}$ Ornstein-Uhlenbeck equation

$$
\partial_{t}^{2} \sigma=-\frac{1}{\mu}\left[k b^{2}+f_{0} N_{p}+\frac{f_{0} \mu N}{\chi}\right] \partial_{t} \sigma-\frac{f_{0} k b^{2} N}{\mu \chi} \sigma+\frac{f_{0} k}{\mu}\left[V\left(N_{p}+\frac{\mu N}{\chi}\right)+\frac{k b^{3} N}{\chi} x(0)\right]-\frac{f_{0} k b}{\mu \chi} \sum_{i=1}^{N_{p}} \eta_{i}
$$

that do not displays serration as seen above. To obtain the Eq16, the relations $\sum_{i=1(i \neq j)}^{N} \sum_{j=1}^{N} 1 /\left(x_{i}-x_{j}\right)=0$ and $\sum_{i=1(i \neq j)}^{N} \sum_{j=1}^{N} \partial_{t}\left(x_{i}-x_{j}\right) /\left(x_{i}-x_{j}\right)^{2}=0$ has been employed.

In the end, from Eqs 12,13 14 we have found four necessary conditions to have serration: i) first of all, the system must be composed by more than one dislocation (as found in Ref. 23] in the low noise limit, and as results in the general case from Eq 11). In other words, serration in the stress response of the system, when present, comes from a collective effect of many interacting dislocations. To verify this, we studied the role of the interaction force between dislocations analysing the average stress $\langle\sigma\rangle$ as a function of time (or strain) for different values of the interaction force itself (obtained changing the value of the shear modulus $G$ ), for parameter values in which serration is observed $(\mu / \chi=0.5$, $1 / T=10^{4}$, see the definition of $T$ below, and $V=0.003$ ). In Fig 1 we displayed the average stress $\langle\sigma\rangle$ (performed on 50 samples) as a function of time obtained integrating numerically Eqs 1213. From it we can see that serration disappears when the strength of the interaction force between dislocations decreases. In particular, for vanishing interaction force $(G=0)$, the stationary average stress is given by the expression: $\langle\sigma\rangle_{s}=V\left[\left(N_{p} / N\right) \chi+\mu\right] \simeq 0.051$, as discussed in the following analysis of Fig.2 ii) dislocations must interact with pinning centers; iii) pinning centers must not be all quenched (see Eq15); iv) dislocations must not be all pinned by impurities (see Eq16). These conditions are all in agreement with the DSA concept.

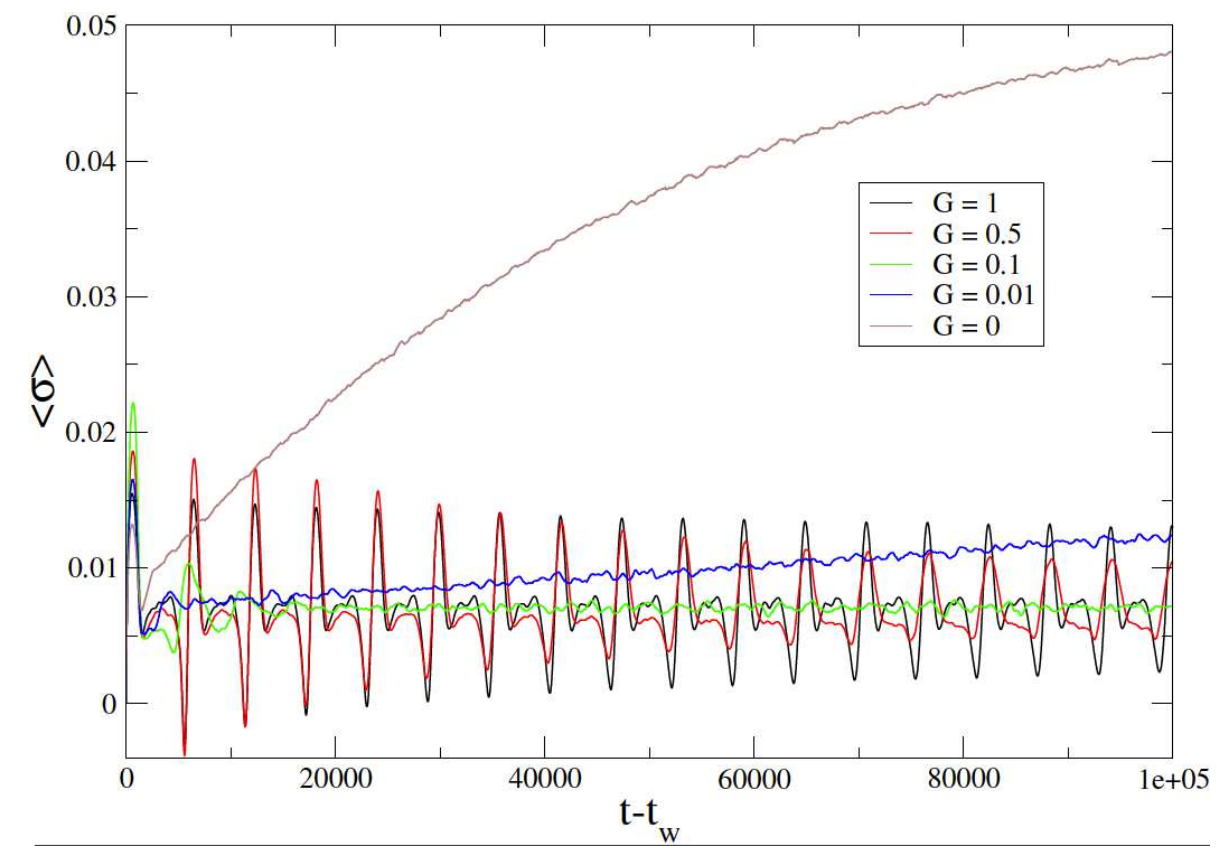

FIG. 1: Average stress $\langle\sigma\rangle$ (performed on 50 samples) as a function of time obtained integrating numerically Eqs 1213 The parameters of the system $\left(\mu / \chi=0.5,1 / T=10^{4}\right.$, and $\left.V=0.003\right)$ are chosen in the range in which serration is observed in order to investigate the role of the interaction force between dislocations changing the value of the shear modulus $G$. From it we can see that serration disappear when the strength of the interaction force between dislocations decreases.

In order to verify the previous four necessary conditions to have serration, and to investigate the behavior of the 
system in the entire free parameter space $(V, \chi, D)$, we integrated numerically Eqs $12[13$ We considered $N=32$ dislocations with an average spacing $d=16$ and average pinning center spacing $d_{p}=L / N_{p}=2$. Because of $L=d N$, is $N_{p}=d N / d_{p}=8 N$. Instead of using the set of parameters $(V, \chi, D)$, we employed this other $(V, \mu / \chi, 1 / T)$ where $T$ is the temperature of the system defined in the following. Although we choose, without less of generality, $\mu=1$ we prefer to keep explicitly the ratio $\mu / \chi$. From the other hand, the explicit introduction of the temperature variable permits to compare the phase diagram of PLC effect (in Fig. 3) with results from literature [29]. The definition of temperature is not something obvious in DDD models. Here we introduce the temperature $T$ of the system as obtained from the Einstein fluctuation-dissipation relation: $T=2 D / \chi$. One difficulty in deriving a general theory of plasticity is due to the presence of thermal as well as athermal dislocation activated processes [13]. For this reason is still lacking a clear definition of temperature in DDD models. The simple one we use here can be considered a good definition for high temperatures and low stresses in which cases diffusional deformation mechanisms become predominant [1]. In other regimes in which this definition of $T$ is not a good approximation, we will discuss how it is related to the behavior of the system.

In Fig. 2 the average stress $\langle\sigma\rangle$, obtained integrating numerically Eqs. 12113, as a function of time $t-t_{w}$ is graphicated for driving velocity $V=0.001,0.003$, mobility ratio $\mu / \chi=0,0.5,1$ and inverse temperature $1 / T \rightarrow \infty$, $1 / T=10^{4}, 2 \cdot 10^{3}, 4 \cdot 10^{2}$. The average $\langle\cdot\rangle$ is performed on 50 samples. In the analysis of Fig. 2 we distinguish two cases, the first one for $\mu / \chi=0$ and the second one for $\mu / \chi>0$. The case $\mu / \chi=0$ corresponds to quenched pinning centers $\left(d x_{s, j} / d t=0\right.$, see Eq. 15). From Fig. 2a,b we see that for $\mu / \chi=0$, the average stress, after oscillations decreasing in time, reach the stationary value $\langle\sigma\rangle_{s}=\lim _{t \rightarrow \infty}\langle\sigma\rangle=V \mu$.

In the case of $\mu / \chi>0$ we expect that at low temperatures and low driving velocity, or at low temperatures and high driving velocity, but high values of mobility ratio, dislocations are pinned by impurities. This is confirmed by Fig. 2r,e,f from which we can see that the stationary value of the average stress is given by the relation $\langle\sigma\rangle_{s}=V\left[\left(N_{p} / N\right) \chi+\mu\right]$, as can be obtained from Eq. 16. To understand the role of dislocation interaction when they are pinned during the whole dynamics, we can consider the Eq. 11] obtained for the dynamics of a single dislocation generalized to the case in which the damping constant of each impurity is $\chi$. Therefore the expression of the stationary external force becomes: $\lim _{t \rightarrow \infty}\langle F\rangle=V\left(N_{p} \chi+\mu\right)$, in which $N_{p}$ is the number of impurities around the only present dislocation. In the case of $N$ dislocations pinned by $N_{p}$ impurities during the whole dynamics, we have that on average each dislocation $i$ is pinned by $N_{p} / N$ impurities (considering that the initial spatial distribution of dislocations and pinning centers is a random flat ones). If we now suppose that in these conditions (obstacles that cannot unpin from dislocations), dislocations do not fluctuate too much around theirs equilibrium positions (that is a configuration of equidistant dislocations), we can conclude that the average stationary stress can be obtained from the formula for the single dislocation pinned by $N_{P} / N$ obstacles case. This means: $\langle\sigma\rangle_{s}=\left\langle F\left(N_{p} \rightarrow N_{p} / N\right)\right\rangle_{s}$ that can be verified employing the previous generalized version of Eq. 11. When $T$ or $V$ increases, the number of impurities that pin dislocations decreases, so $\langle\sigma\rangle_{s}$ decreases.

From Fig. 2 we can see that stationary fluctuations in the stress (serration) emerge for the values parameter $V=0.003, \mu / \chi=0.5$ and $1 / T=10^{4}$. In Fig. 2 results for driving velocities higher than $V=0.003$ and temperatures $T$ smaller than $10^{-4}$ are not reported because in these regimes we reach the limit of our model that continue to give serration in the stationary average stress, while in real systems we should not have serration [13, 29]. Negative values of stress fluctuations at earliest times correspond to a sudden increases in the dislocations average position (see Eq. (13) that happens when dislocations escape from many pinning centers. Indeed, when it happens, we can observe this effect at earliest times of the dynamics (for $t>t_{w}$ ), because the absence of the external stress for $0<t<t_{w}$ permits to dislocations to accumulate pinning centers.

In order to summarise the results obtained from the present model and to compare them with experimental 29] and others theoretical approache [13], we depicted in Fig. 33 relying on data displayed in Fig. 2 and others data not displayed there, a phase diagram for the PLC effect in the parameter space $(1 / T, V)$ for $\mu / \chi=0.5$. In the range of parameters inside the grey region, the PLC effect takes place. The dashed red lines indicate where the model described by Eqs. $12[13$ start to fail in predicting the PLC effect. In particular, for high driving velocities new dislocation mechanisms, as dislocation multiplication, climbing and others complex behaviors, must be taken into account. Considering these mechanisms, serration must disappear for high driving velocities irrespective of the other parameter values. While in the case of small temperatures, non thermal dislocation processes become relevant in relation to the thermal ones, and the Einstein fluctuation-dissipation relation do not holds anymore. Also in this case, considering the presence of non thermal dislocation processes, serration must disappear for small temperatures irrespective of the other parameter values. 

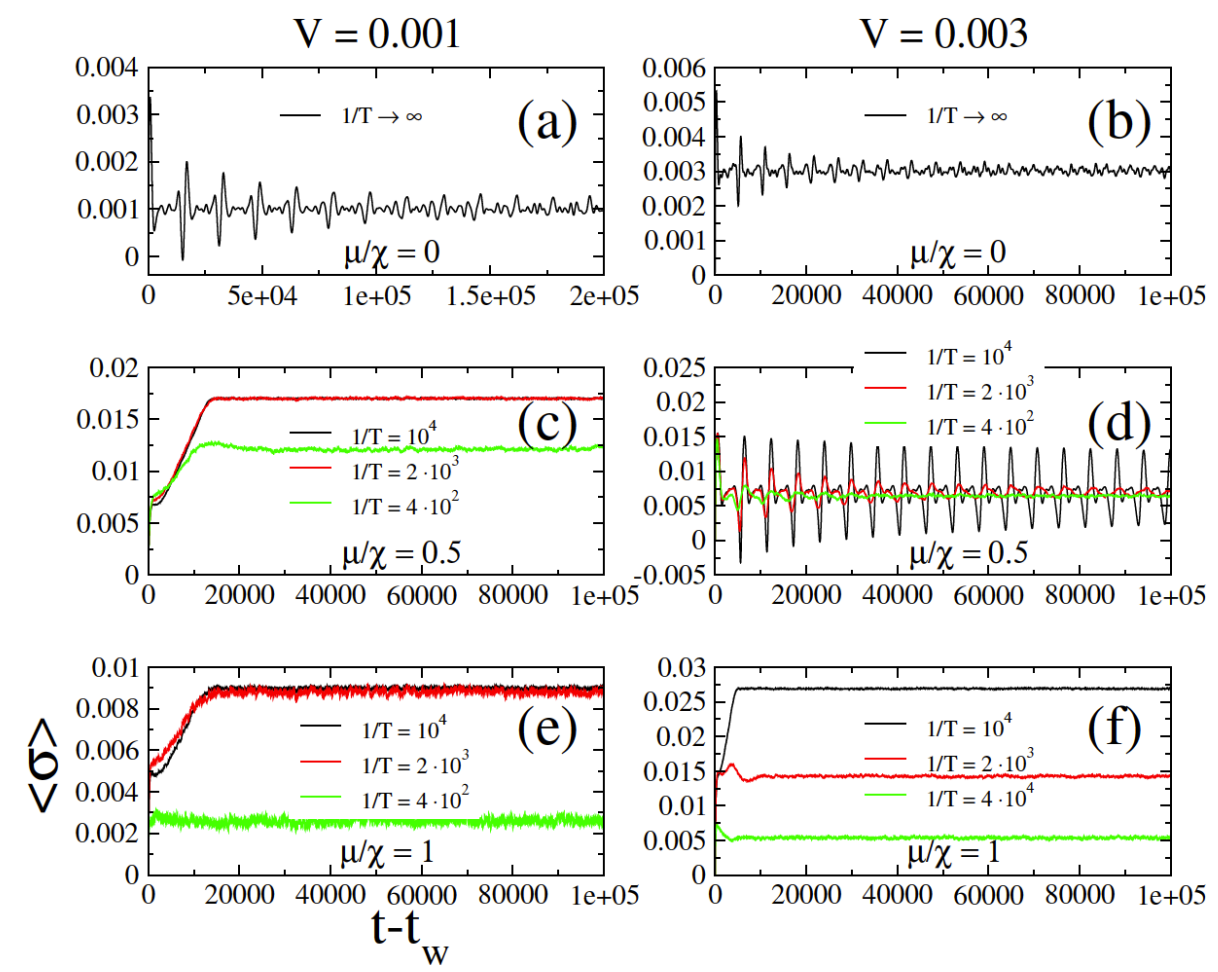

FIG. 2: Average stress $\langle\sigma\rangle$, obtained integrating numerically Eqs. 12]13 as a function of time $t-t_{w}$, for $t_{w}=10^{6} \cdot d t=10^{4}$, driving velocity $V=0.001,0.003$, mobility ratio $\mu / \chi=0,0.5,1$ and inverse temperature $1 / T \rightarrow \infty, 1 / T=10^{4}, 2 \cdot 10^{3}, 4 \cdot 10^{2}$.

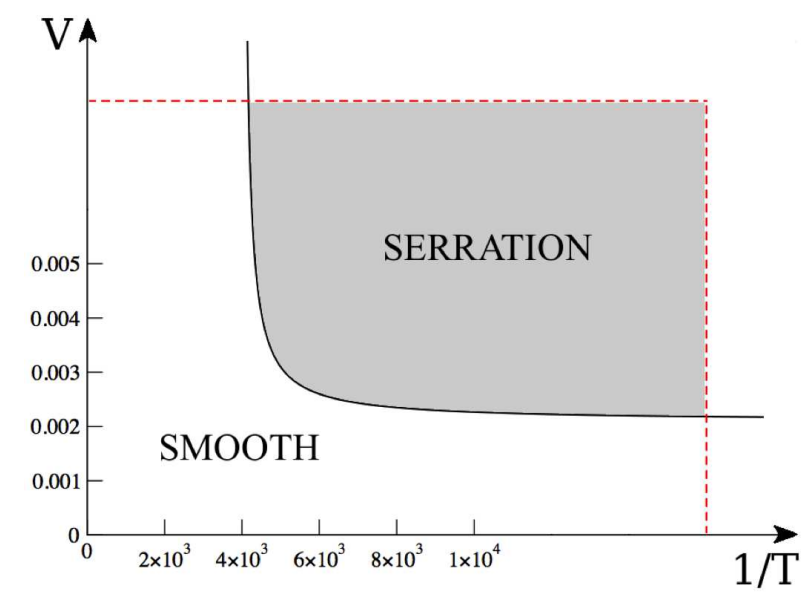

FIG. 3: Semi-quantitative phase diagram for the PLC effect in the parameter space $(1 / T, V)$ for $\mu / \chi=0.5$. In the range of parameters inside the grey region, the PLC effect take place (i. e. the average stress $\langle\sigma\rangle$ displays serration). The dashed red lines indicate where the model described by Eqs. 12]13 start to fail in predicting the PLC effect.

\section{THE RELEVANT TIME SCALES IN SERRATION}

The behavior of the system described by Eqs. 12/13/14 can be analyzed in terms of time scales. The fundamental time scales are: i) the capturing time $t_{c}$ that accounts for the average time needed for a dislocation to capture a pinning center; ii) the aging time $t_{a}$ that accounts for the average time needed for a dislocation to escape from a pinning center. In order to compute these times we consider that a pinning center pins a dislocation if the distance between them is smaller then $\xi_{c}=3 \xi_{P}=3$ (otherwise the attraction force between them is considered negligible). In Tab. I we report the time ratio $t_{a} / t_{c}$ for the same values of driving velocity $V$, mobility ratio $\mu / \chi$, and inverse 
TABLE I: Time scales ratio: $t_{a} / t_{c}$. The computational error is one over the last digit.

\begin{tabular}{|c|c|c|c|c|c|}
\hline \multicolumn{3}{|c|}{$V=0.001$} & \multicolumn{3}{|c|}{$V=0.003$} \\
\hline$\mu / \chi=0$ & 0.60 & $\leftarrow$ for all $1 / T$ & $\mu / \chi=0$ & 0.60 & $\leftarrow$ for all $1 / T$ \\
\hline & 65.07 & $\leftarrow 1 / T=10^{4}$ & & 0.75 & $\leftarrow 1 / T=10^{4}$ \\
\hline$\mu / \chi=0.5$ & 66.48 & $\leftarrow 1 / T=2 \cdot 10^{3}$ & $\mu / \chi=0.5$ & 0.75 & $\leftarrow 1 / T=2 \cdot 10^{3}$ \\
\hline & 4.09 & $\leftarrow 1 / T=4 \cdot 10^{2}$ & & 0.64 & $\leftarrow 1 / T=4 \cdot 10^{2}$ \\
\hline & 78.73 & $\leftarrow 1 / T=10^{4}$ & & 146.97 & $\leftarrow 1 / T=10^{4}$ \\
\hline$\mu / \chi=1$ & 37.93 & $\leftarrow 1 / T=2 \cdot 10^{3}$ & $\mu / \chi=1$ & 2.03 & $\leftarrow 1 / T=2 \cdot 10^{3}$ \\
\hline & 1.13 & $\leftarrow 1 / T=4 \cdot 10^{2}$ & & 0.79 & $\leftarrow 1 / T=4 \cdot 10^{2}$ \\
\hline
\end{tabular}

temperature $1 / T$ for which the average stress $\langle\sigma\rangle$ has been computed and displayed in Fig. 2. First of all, we can observe that for $V \rightarrow \infty$ or $\mu / \chi \rightarrow 0$ or $1 / T \rightarrow 0$, the time ratio become: $t_{a} / t_{c}=\left(2 \cdot \xi_{c}\right) /\left(d-2 \cdot \xi_{c}\right)=0.6$ where $d$ is the dislocation average interdistance. Indeed, in these conditions we can consider the pinning centers to be fixed respect to dislocations (or vice versa) during the dynamics and the ratio $t_{a} / t_{c}$ become nothing more than the ratio between the average (in time) length per dislocation and pinning center over which dislocations are considered pinned (that is $\alpha\left(t_{a v}\right) \cdot 2 \cdot \xi_{c}$ ) and that over which they are not (that is $\alpha\left(t_{a v}\right) \cdot\left(d-2 \cdot \xi_{c}\right)$ ) where $\alpha\left(t_{a v}\right)$ is the same parameter for the two lengths and depends only on the time $t_{a v}$ over which the average is performed. Looking at the values displayed in Tab! we can observe that in general the time ratio $t_{a} / t_{c}$ decreases significantly as $V$ increases, or as $\mu / \chi$ or $1 / T$ decreases, but only for $\mu / \chi=0.5$ and $V=0.003$ we have that $t_{a} / t_{c}$ remains small (bigger than, but near, the value 0.6 ) for changing $1 / T$. In particular, for $\mu / \chi=0.5, V=0.003$ and $1 / T=10^{4}, 2 \cdot 10^{3}$, the two times $t_{a}$ and $t_{c}$ (which ratio is displayed in bold on Tab【) are of the same order of magnitude, but the pinning centers, for these parameter values, are not fixed respect to dislocations (or vice versa).

In the end we can conclude that for values of parameters $V, \mu / \chi$ and $1 / T$ for which serration is observed, we have that the two relevant times $t_{a}$ and $t_{c}$ are of the same order of magnitude (remembering that the value 0.6 corresponds to a special case in our model). This is in agreement with phenomenological models [13] and represents a link between microscopic DDD model parameters (appearing in Eqs.1211314) from which the quantities $t_{a}$ and $t_{c}$ can be computed, and macroscopic quantities like $V, \mu / \chi$ and $T$.

\section{SPATIO-TEMPORAL DISTRIBUTION OF DISLOCATIONS AND IMPURITIES}

Analysing spatio-temporal distributions of pinning centers and dislocations can help to better understand and unify previous considerations and results. First of all, we computed the average pinning centers distribution $\rho_{p . c .}$. averaging each distribution of pinning centers around every dislocation, and averaging over 50 different realizations of the dynamics. In the lower part of Fig. 4 we display average pinning center distributions around dislocations for different times that correspond to different value of the average stress (which values are indicated in the upper stress vs time graphic of the same figure). The more interesting case, reported in Fig,4 and corresponding to Fig 22(d), is that for which changing temperature causes serration appearance or disappearance (that is for $\mu / \chi=0.5, V=0.003$ ).

The distributions $\rho_{\text {p.c. }}$ are unnormalized so that the average number of pinning centers that pins dislocations are obtained by the integral: $\int_{-\xi_{c}}^{\xi_{c}} \rho_{\text {p.c. }}\left(x_{\text {p.c. }}\right) d x_{\text {p.c. }}=\left\langle N_{p}\right\rangle$. To understand what happens when serration in the stress appears, we reported in Fig. 4 the distributions in correspondence of stress drop, bump and re-drop. For temperatures for which the stress do not develops serration (for $1 / T=4 \cdot 10^{2}, 2 \cdot 10^{3}$ ), the distribution shape of pinning centers around dislocations do not change in time and displays a peak more or less wide and narrow depending on the values of $V, \mu / \chi$ and $1 / T$. For temperatures for which serration develops (for $1 / T=10^{4}$ ), the distributions change in a way that when pinning centers reach dislocations from the right side (indeed $V=0.003>0$ ), Fig. \&(b), and start to pin them, stress drops because dislocations are accelerated (see Eq. 13), then after a while dislocation velocities go down because they are pinned (when the corresponding distribution has developed a wide narrow peak), Fig. 廿4(c),(d). Finally, when dislocations are able to depin and then their velocities start to increase again, the stress re-drops, Fig. 4(e),(f). Above, analysing the stationary value of the stress (Fig. 21), we argued that at low temperatures (in Fig.2(c),(e),(f)) dislocations are completely pinned, and verified that by means of the formula $\langle\sigma\rangle_{s}=V\left[\left(N_{p} / N\right) \chi+\mu\right]$ previously obtained from the equations of motion imposing the condition of complete pinning. Integrating the distributions of pinning centers, $\rho_{\text {p.c. }}$, for low temperatures, we can obtain the average number of pinning centers around dislocations that is $\left\langle N_{p}\right\rangle=N_{p} / N=8$ which confirms directly that in these cases all pinning centers are pinning dislocations. Computing the standard deviation of the average distribution in Fig 4 confirms that when serration emerges, for $1 / T=10^{4}$, dislocations depin in a choerent way. Indeed, in this case the standard deviation is big near the peak of the distribution, while is quite small away from it. In the cases in which serration is not observed, for $1 / T=10^{4}, 4 \cdot 10^{2}$, 

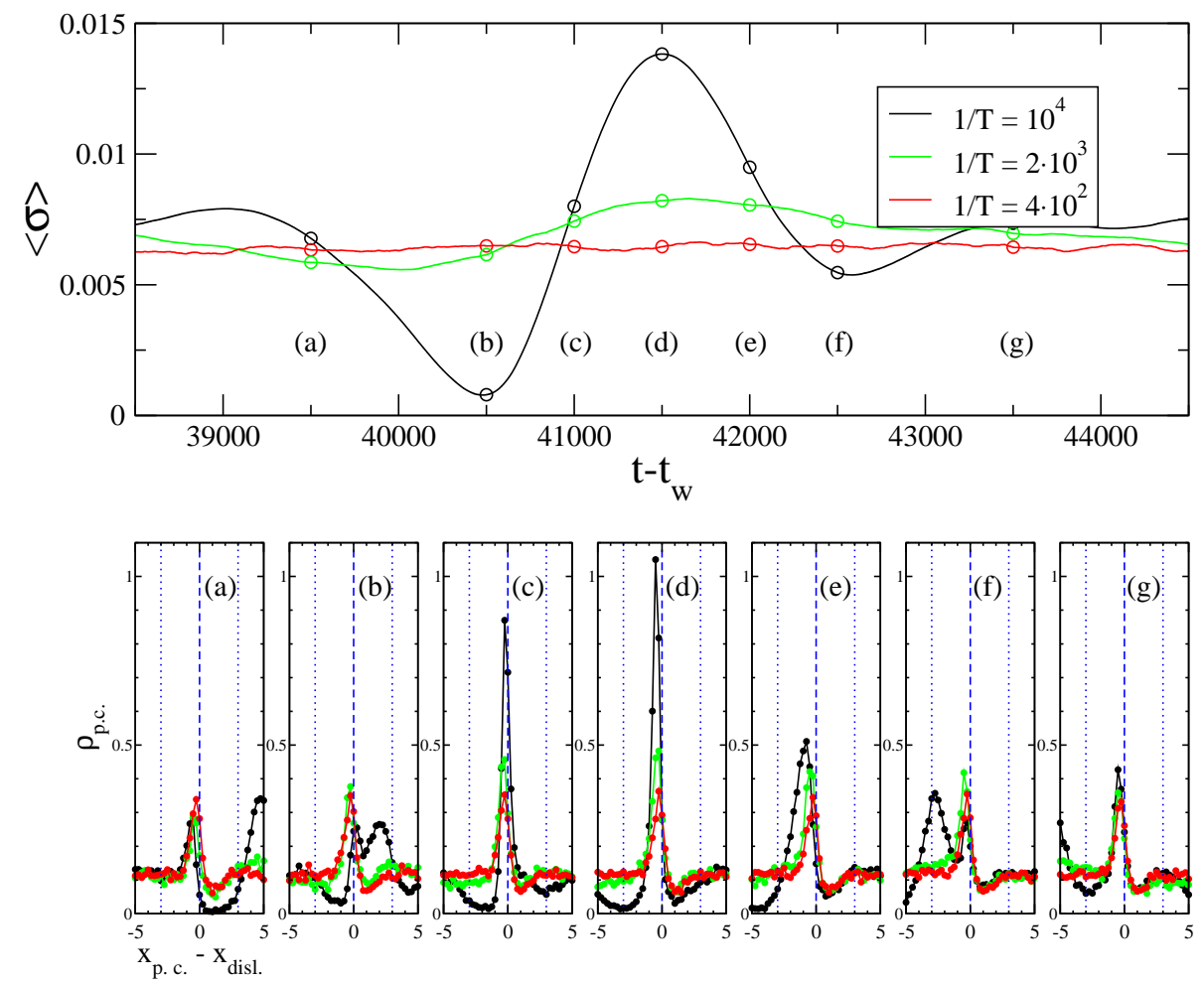

FIG. 4: Spatio-temporal distribution of pinning centers around dislocations. The distributions are obtained averaging for a specific time the different distributions around every dislocation, and averaging over 50 different realizations of the dynamics. In the lower par of the figure are indicated with $x_{p . c .}$ the pinning centers position and with $x_{d i s l}$. the dislocation position. The vertical dashed blue line indicate the dislocation position and the two vertical dotted blue lines indicate the cutoff $\xi_{c}$ of the dislocation-pinning centers interaction. In the upper part of the figure, the average stress for a specific time region is indicated. The corresponding complete curve is reported in Fig. 2 .

the standard deviation is almost constant in time and space. Considering this result and that, in particular for $1 / T=4 \cdot 10^{2}$, the time ratio $t_{a} / t_{c}$ (see Tab. I) is the same as for the case $1 / T=10^{4}$, and that the average curve is constant in time, we can conclude that when serration disappear, this means that dislocations depin in a incoherent way (at different times).

In Fig. 5 we can see a representative portion of dislocations and pinning centers motion in the case in which serration is not present (a), and that in which it appears (b). The dark lines are dislocations. They have a slope equal to $V=0.003$, unless small deviations in correspondence of pinning centers (the green lines) pinning dislocations. In Fig. $5 \mathrm{~b}$ the vertical dotted and dashed red lines correspond to times for which the average stress has a minimum and a maximum respectively. The minimum in the average stress correspond to many dislocations that are pinned, while the successive maximum to many dislocations that depin. These processes are also present at intermediate times, that cause the appearance of small oscillations in the average stress between a big peak and a big valley (see Fig. 1 and Fig. 2(d)). The dashed blu line, $y \sim v_{\text {drift }} t$, indicates the drift of pinning centers that move with velocity $v_{\text {drift }} \simeq 2.5 \cdot 10^{-4}$. This drift is caused by the interaction with dislocations.

In Fig. [6 we can see the entire dynamics of all dislocations in a case in which there is not serration (a), and another in which it appears (b). In particular, the term $V t$ is subtracted to dislocation positions, so we have horizontal lines, while in Fig. 5 we have lines with a slope equal to $V$. Moreover, the position of dislocations are rescaled in order to have that the average inter-distance between them is 0.5 instead of $d=16$. This last rescaling has been done to evidence the fluctuations of dislocations position. Like in Fig. 5 , the vertical dotted and dashed red lines correspond to times for which the average stress has a minimum and a maximum respectively. From Fig. 6b we can see essentially two interesting things: minimum and maximum values in the rescaled dislocations position correspond to maximum and minimum values in the average stress respectively; and the fluctuation profile of the position of one dislocation (in correspondence of serration) can propagate with a velocity of $V-v_{\text {drift }}$. This last point means that when pinning centers start to clustering, they remain usually clustered during the entire dynamics. The dashed blue line $y \sim-0.5 /\left[d /\left(V-v_{\text {drift }}\right)\right] x=-0.875 \cdot 10^{-4} x$ in Fig. [6 indicate this behavior of pinning centers. 

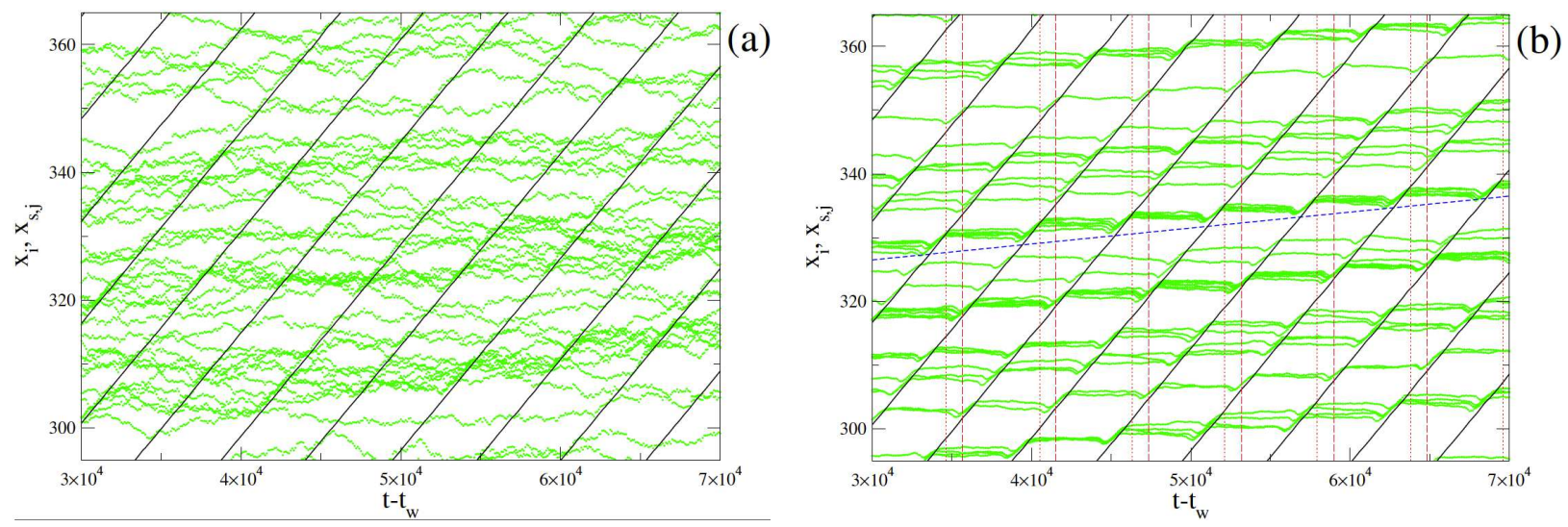

(b)

FIG. 5: Representative portion of dislocations and pinning centers motion for a single realization of the dynamics for parameter values: $V=0.003, \mu / \chi=0.5$ and $1 / T=4 \cdot 10^{2}(\mathrm{a}), 1 / T=10^{4}(\mathrm{~b})$. The dark lines with a slope equal to $\mathrm{V}=0.003$ are dislocations, and the green lines are pinning centers. (b) The vertical dotted and dashed red lines correspond to times for which the average stress has a minimum and a maximum respectively. The dashed blu line $y \sim v_{\text {drift }} t$ indicates the drift of pinning centers that move with velocity $v_{\text {drift }} \simeq 2.5 \cdot 10^{-4}$.
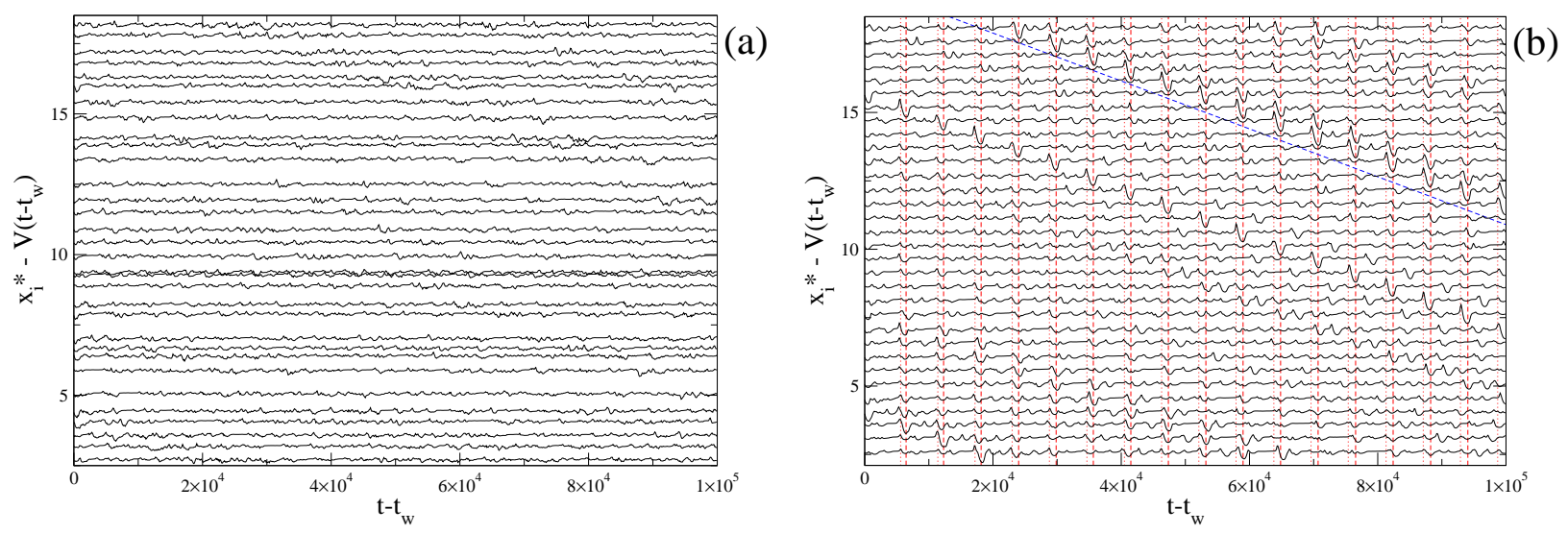

FIG. 6: Dynamics of all dislocations for a single realization of the dynamics for parameter values: $V=0.003, \mu / \chi=0.5$ and $1 / T=4 \cdot 10^{2}$ (a), $1 / T=10^{4}(\mathrm{~b})$. To evidence fluctuations, dislocations position, at which the term $V t$ has been subtracted, are rescaled in a way that the average inter-distance between them is 0.5 instead of $d=16$. (b) The dashed blue line $y \sim-0.5 /\left[d /\left(V-v_{d r i f t}\right)\right] x=-0.875 \cdot 10^{-4} x$ indicate clustering properties of pinning centers, as discussed in the text.

In Fig. 7 we display the superposition of fluctuations in the position of all dislocations, $\Delta x_{i}$, in a case in which there is no serration (a) and another in which it appears (b). These fluctuations has been obtained as: $\Delta x_{i}=$ $\left(x_{i}-V t\right)-\left\langle\left(x_{i}-V t\right)\right\rangle_{t}$, where the time average is performed on the entire dynamics. We also display the average of all $\Delta x_{i}$ with the relative standard deviation, and the vertical dotted and dashed lines correspond to times for which the average stress has a minimum and a maximum respectively. We can see another time that the minimum and maximum values of the average fluctuation position correspond to the maximum and minimum in the average stress respectively.

\section{DISCUSSION}

We investigated the dynamics of a dislocation assembly interacting with mobile impurities studying the case of a one-dimensional dislocation pileup. In order to connect this model to the PLC effect, we studied the stress response of the system under an external constant strain rate. The free parameters of the system have been reduced to the driving velocity $V$, that controls the imposed constant strain rate, the mobility ratio $\mu / \chi$ between dislocations and mobile impurities, and the temperature $T$. To this end, we have employed an effective definition of temperature that should be valid except for low temperatures and high stresses. Analysing the average stress of the system $\langle\sigma\rangle$ in the 

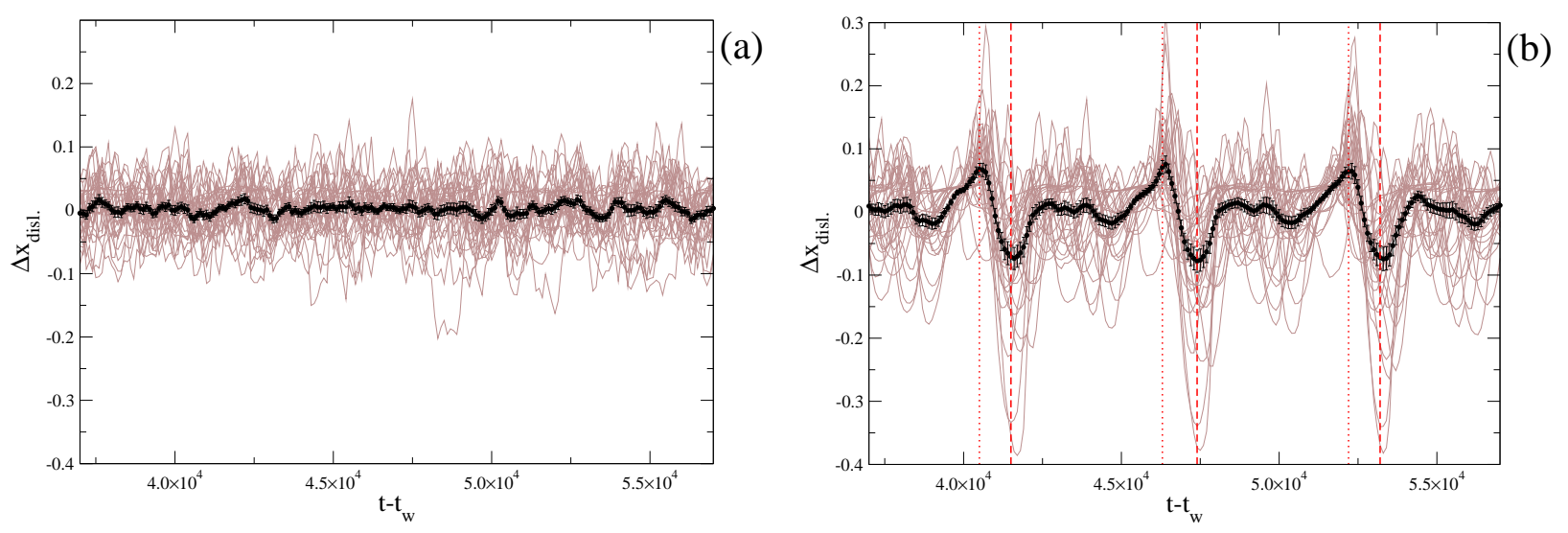

FIG. 7: In brown we display the superposition of the position fluctuations of all dislocations, $\Delta x_{i}=\left(x_{i}-V t\right)-<\left(x_{i}-V t\right)>_{t}$ where the positions $x_{i}$ are reported in Fig. 6, and the time average is performed on the entire dynamics. In dark we can see the average of all $\Delta x_{i}$ with the relative standard deviation. (b) The vertical dotted and dashed lines correspond to times for which the average stress has a minimum and a maximum respectively.

parameter space $(V, \mu / \chi, 1 / T)$, we found a region characterized by stationary fluctuations in the stress (serration) (see Fig. 3).

The interpretation for the onset of serration in the present model agrees with the general concept of DSA but takes explicitly into account the role of dislocation mutual interactions. The emergence of serration corresponds to the situation in which impurities diffuse at a rate that allows them to pin dislocations (i.e. the capturing time $t_{c}$ is not too high). At the same time dislocations should be able to escape from pinning centers after an aging time $t_{a}$ which implies that $t_{a}$ is not too high, otherwise when dislocations unpin, they would move at large speed until they are arrested again and pinning centers will not be able to reach them, but also not too small, because in this case pinning centers will not be able to pin dislocations. Serration correspond to the case in which the two characteristic time scales, $t_{a}$ and $t_{c}$, are of the same order of magnitude (see Tab. 【).

From another point of view, the origin of serration, in a specific range of parameter values $(V, \mu / \chi, T)$, is due to the localization of impurities in a limited number of clouds under the action of dislocation induced stresses and to the possibility for dislocations to escape from their pinning clouds without randomizing excessively the spatial distribution of impurities. The spatial localization of pinning centers is only possible due to the coherent action of several interacting dislocations. If we randomize the interaction between dislocations, for instance by choosing Burgers vectors $b_{i}$ for each dislocation $i$ from a random distribution, serration disappears. Spatial randomization of pinning centers results in an incoherent contribution to the total stress fluctuations of each dislocation and in the impossibility to form clouds of pinning centers and therefore in the disappearance of serration. Only when the contribution of each dislocation to the total stress fluctuations are coherent, serration is observed.

In order to extend the validity of the definition of temperature and generalize the present model, the following effects could be introduced: i) a viscosity therm for dislocations; ii) pinning centers with different mobilities. The last modification could give different type of serration like in experiments in which three types of bands can be identified (type A,B,C) [13].

\section{Acknowledgments}

SZ is supported by the European Research Council through the Advanced Grant SIZEFFECTS.

[1] M. Zaiser, Adv. Phys. 55, 185 (2006).

[2] J. Weiss and J. R. Grasso, J. Phys. Chem. B 101, 6113 (1997).

[3] M.-C. Miguel, A. Vespignani, S. Zapperi, J. Weiss, and J. R. Grasso, Nature 410, 667 (2001).

[4] M.-C. Miguel and A. Vespignani and M. Zaiser and S. Zapperi, Phys. Rev. Lett. 89, 165501 (2002).

[5] M. Z. Butt and P. Feltham, J. Mater. Sci. 28, 2557 (1993).

[6] H. Neuhäuser, Phys. Scr., T 49, 412 (1993). 
[7] F. Leoni and S. Zapperi, Phys. Rev. Lett. 102, 115502 (2009).

[8] J. W. Cahn, Acta Metall. 10, 789 (1962).

[9] D. Blavette, E. Cadel, A. Fraczkiewicz, and A. Menand, Science 286, 2317 (1999).

[10] E. M. Chudnovsky, Europhys. Lett. 43, 445 (1998).

11] A. Portevin and F. Le Chatelier, C. R. Acad. Sci. Paris 176, 507 (1923).

[12] A.H. Cottrell and B.A. Bilby, Proc. R. Soc. London Ser. A 62, 49 (1949); A.H. Cottrell, Philos. Mag. 44, 829 (1953).

[13] G. Ananthakrishna, Phys. Rep. 440, 113 (2007).

[14] H. Neuhäuser, in: Dislocations in Solids (Edited by F. R. N. Nabarro, North-Holland, Amsterdam, 1983).

[15] V. Mohles, Mater Sci. Eng. 309-310, 265 (2001).

[16] G. Monnet, Philos. Mag. 86, 5927 (2006).

[17] F. F. Csikor, C. Motz, D. Weygand, M. Zaiser, and S. Zapperi, Science 318, 251 (2007).

[18] B. Devincre, T. Hoc, and L. Kubin, Science 320, 1745 (2008).

[19] J. Senger, D. Weygand, P. Gumbsch, and O. Kraft, Scr. Mater. 58, 587 (2008).

[20] B. Liu, D. Raabe, P. Eisenlohr, F. Roters, A. Arsenlis, and G. Hommes, Acta Mater. 59, 7125 (2011).

[21] G. P. Reddy, C. Robertson, C. Depres, and M. Fivel, Acta Mater. 61, 5300 (2013).

[22] C. Bairnsfather, C. J. Olson Reichhardt and C. Reichhardt, Europhys. Lett. 94, 18001 (2011).

[23] L. Laurson and M. J. Alava, J. Stat. Mech. P07003 (2008).

[24] H. Risken, The Fokker-Plank Equation (Springer Verlag, 1984).

[25] M. F. Kanninen and A. R. Rosenfield, Philos. Mag. 20, 569 (1969).

[26] J. P. Hirth and J. Lothe, Theory of Dislocations (Wiley \& Sons, 1982).

[27] B. Marcos and T. Baertschinger, M. Joyce, A. Gabrielli, and F. Sylos Labini, Phys. Rev. D 73, 103507 (2006).

[28] F. J. Dyson, J. of Math. Phys. 3, 1191 (1962).

[29] M. Lebyodkin, L. Dunin-Barkovskii, Y. Bréchet, Y. Estrin, and L. P. Kubin, Acta Mater. 48, 2529 (2000). 